\title{
Characterization of transverse beam emittance of electrons from a laser-plasma wakefield accelerator in the bubble regime using betatron $x$-ray radiation
}

\author{
S. Kneip, ${ }^{1,2}$ C. McGuffey, ${ }^{2}$ J. L. Martins, ${ }^{3}$ M. S. Bloom, ${ }^{1}$ V. Chvykov, ${ }^{2}$ F. Dollar, ${ }^{2}$ R. Fonseca,,${ }^{3,4}$ S. Jolly, ${ }^{5}$ \\ G. Kalintchenko, ${ }^{2}$ K. Krushelnick, ${ }^{2}$ A. Maksimchuk, ${ }^{2}$ S. P. D. Mangles, ${ }^{1}$ Z. Najmudin, ${ }^{1}$ C. A. J. Palmer, ${ }^{1}$ K. Ta Phuoc, ${ }^{6}$ \\ W. Schumaker, ${ }^{2}$ L. O. Silva, ${ }^{3}$ J. Vieira, ${ }^{3}$ V. Yanovsky, ${ }^{2}$ and A. G. R. Thomas ${ }^{2}$ \\ ${ }^{1}$ The Blackett Laboratory, Imperial College London, London, SW7 2BZ, United Kingdom \\ ${ }^{2}$ Center for Ultrafast Optical Science, University of Michigan, Ann Arbor, Michigan 48109, USA \\ ${ }^{3}$ GoLP/Instituto de Plasmas e Fusão Nuclear, Laboratório Associado, Instituto Superior Técnico, Lisbon, 1049-001, Portugal \\ ${ }^{4}$ DCTI, ISCTE, Lisbon University Institute, Lisbon, 1649-026, Portugal \\ ${ }^{5}$ Department of Physics \& Astronomy, University College London, London, WC1E 6BT, United Kingdom \\ ${ }^{6}$ Laboratoire d'Optique Appliquée, ENSTA ParisTech-CNRS UMR7639, Ecole Polytechnique, 91761 Palaiseau, France
}

(Received 30 March 2011; published 2 February 2012)

\begin{abstract}
We propose and use a technique to measure the transverse emittance of a laser-wakefield accelerated beam of relativistic electrons. The technique is based on the simultaneous measurements of the electron beam divergence given by $v_{\perp} / v_{\|}$, the measured spectrum $\gamma$, and the transverse electron bunch size in the bubble $r_{\perp}$. The latter is obtained via the measurement of the source size of the $\mathrm{x}$ rays emitted by the accelerating electron bunch in the bubble. We measure a normalized rms beam transverse emittance $<0.5 \pi \mathrm{mm}$ mrad as an upper limit for a spatially Gaussian, spectrally quasimonoenergetic electron beam with $230 \mathrm{MeV}$ energy in agreement with numerical modeling and analytic theory in the bubble regime.
\end{abstract}

DOI: 10.1103/PhysRevSTAB.15.021302

PACS numbers: 52.38.Kd, 52.38.Ph, 52.70.La

A laser-wakefield accelerator [1] uses an intense laser pulse to generate a plasma wave, known as a wakefield, with a phase velocity close to the speed of light. If a relativistic electron beam can be inserted copropagating with the wakefield, it can remain in an accelerating phase of the wave for a long distance, and extract substantial energy from the longitudinal electric field. Since plasma waves can support electric fields many orders of magnitude stronger than those in radio-frequency (rf) cavities, plasma accelerators may provide a compact alternative to conventional $\mathrm{rf}$ linear accelerators in the future.

In the nonlinear regime of laser-wakefield acceleration (LWFA), the laser pulse expels electrons from its focal volume [2] but has a negligible effect on the remaining ions, resulting in a bare ion cavity with strong electromagnetic fields. Electrons from the periphery of the cavity are pulled inwards by the strong Coulomb attraction and form a thin high-density sheath around an approximately spherical "bubble" [3,4]. This has ideal linear accelerating and focusing properties for electrons within the cavity and at the point where the sheaths cross at the rear of the bubble, the field is particularly strong. Here, electrons from the plasma can be accelerated to the phase velocity of the bubble in a time shorter than their crossing time and are therefore trapped. Under certain conditions, the

Published by the American Physical Society under the terms of the Creative Commons Attribution 3.0 License. Further distribution of this work must maintain attribution to the author(s) and the published article's title, journal citation, and DOI. self-trapped electron beam can have a quasimonoenergetic energy distribution [5-7].

Most applications require the electron beam to be transported over long distances and/or to be focused into a small space with a minimum of divergence, for example to form high-resolution images. A good measure of the quality of a particle beam is its density distribution in 6D phase space $\left(x, y, z, p_{x}, p_{y}, p_{z}\right)$, which is a characteristic of the beam. The Liouville theorem states that for noninteracting particles, subject to a Hamiltonian (the electromagnetic fields of the plasma) the extent of the beam in phase space $\Gamma$, called emittance, is conserved. For practical purposes the emittance is split into 2D subspaces $x, p_{x}, y, p_{y}$ and $z, p_{z}$. If the area occupied by the beam in the $\left(r_{\perp}, p_{\perp}\right)$ plane is $\Gamma^{\perp}$ with $\perp=x, y$, then the normalized or invariant (transverse) emittance is defined

$$
\epsilon_{n}^{\perp}:=\Gamma^{\perp} /\left(\pi m_{e} c\right),
$$

where $m_{e}$ is the electron mass and $c$ is the speed of light. The gradients of the trajectory in the $\left(r_{\perp}, z\right)$ plane are more easily measured. This motivates the introduction of the trace space $\left(r_{\perp}, r_{\perp}^{\prime}\right)$ where $r_{\perp}^{\prime}=d r_{\perp} / d z=v_{\perp} / v_{\|}$. If the area occupied by the beam in trace space is $A^{\perp}$, then the geometric emittance is defined as

$$
\epsilon^{\perp}:=A^{\perp} / \pi \text {. }
$$

One can show that normalized and geometric emittance are related via $\epsilon_{n}^{\perp}=\epsilon^{\perp} \gamma \beta_{z}=\gamma \beta_{z} \int r_{\perp}^{\prime} d r_{\perp} \leq \gamma \beta_{z} r_{\perp}^{\prime} r_{\perp}$, where $\beta_{z}=v_{z} / c$. The measurement of the normalized emittance $\epsilon_{n}^{\perp}$ reduces to a measurement of the beam energy $\gamma_{\|}=p_{z} /\left(m_{e} c\right) \lesssim \gamma$, the transverse extent $r_{\perp}$, 
and divergence of the beam $r_{\perp}^{\prime}$, where $\beta_{z} \simeq 1$ is assumed for relativistic beams.

The extent in $r_{\perp}$ space of a beam is relatively easy to obtain in general, e.g., by imaging its size with a scintillating screen. The extent in gradient $r_{\perp}^{\prime}$ space of a beam is more difficult to determine in general. For a lower energy electron beam, one standard measurement technique is the "pepper-pot" technique [8,9]. A lead mask consisting of a series of small apertures, significantly smaller than the incident beam profile, allows the extent in $r_{\perp}$ space to be measured and to be related to the angular divergence $r_{\perp}^{\prime}$ of the resulting beamlets at the aperture by assuming ballistic trajectories. This technique was applied to $\simeq 100 \mathrm{MeV}$ electron beams from a laser-wakefield accelerator [10-12] measuring normalized transverse emittances of order $\pi \mathrm{mm}$ mrad. However, it is difficult to extend this method to the $\mathrm{GeV}$ energy monoenergetic beams of recent laserwakefield experiments $[13,14]$ as, in addition to the difficulty of stopping $\mathrm{GeV}$ electrons, the spatial profile of the beam is small and therefore difficult to aperture. The difficulty of measuring the electron energy $\gamma$ simultaneously with $\left(r_{\perp}, r_{\perp}^{\prime}\right)$ is a further disadvantage of the "pepper-pot" technique. The precision of the "pepper-pot" technique is ultimately limited by conflicting design considerations [15]. Another technique to establish the emittance (particularly at conventional radio-frequency accelerators) is based on changing the parameters of magnetic beam line elements such as of a quadrupole lens [15]. Such quadrupole scans are applicable to multi-GeV beams but require an electron beam stability not easily achievable with a LWFA.

In this paper, we propose and use a technique that allows the simultaneous measurement of $\left(\gamma, r_{\perp}, r_{\perp}^{\prime}\right)$ and hence determination of an upper limit to the normalized emittance $\epsilon_{n}^{\perp}$ on a single shot. The electron energy $\gamma$ is measured with a spectrometer, the divergence $r_{\perp}^{\prime}$ is obtained from the distribution in the direction perpendicular to the dispersion, and the beam size $r_{\perp}$ is inferred from the betatron x-ray source size emitted by the electron bunch. Using this technique, we show that the normalized emittance of a $230 \mathrm{MeV}$ electron beam from a LWFA can be $<0.5 \pi \mathrm{mm}$ mrad and agrees with the density scaling in the bubble regime and numerical modeling. Our method will work without modification to measure the emittance of multi-GeV beams anticipated in the near future.

The experiments were performed at the Hercules laser of the Center for Ultrafast Optical Science at the University of Michigan, Ann Arbor. Horizontally, linearly polarized pulses with a central wavelength of $\lambda_{L}=800 \mathrm{~nm}$, a Gaussian full-width half maximum (FWHM) pulse duration of $t_{L}=(32 \pm 2) \mathrm{fs}$, and an energy of $\mathcal{E}_{L}=(2.2 \pm$ $0.1) \mathrm{J}$ were focused to a peak intensity of $I_{0}=$ $(2.0 \pm 0.4) \times 10^{19} \mathrm{~W} \mathrm{~cm}^{-2}$ or an $a_{0}=(3.0 \pm 0.3)$ with an off-axis parabolic mirror of focal length $f=1 \mathrm{~m}$ and an $F$ number of $F=10$. The transverse profile of the laser

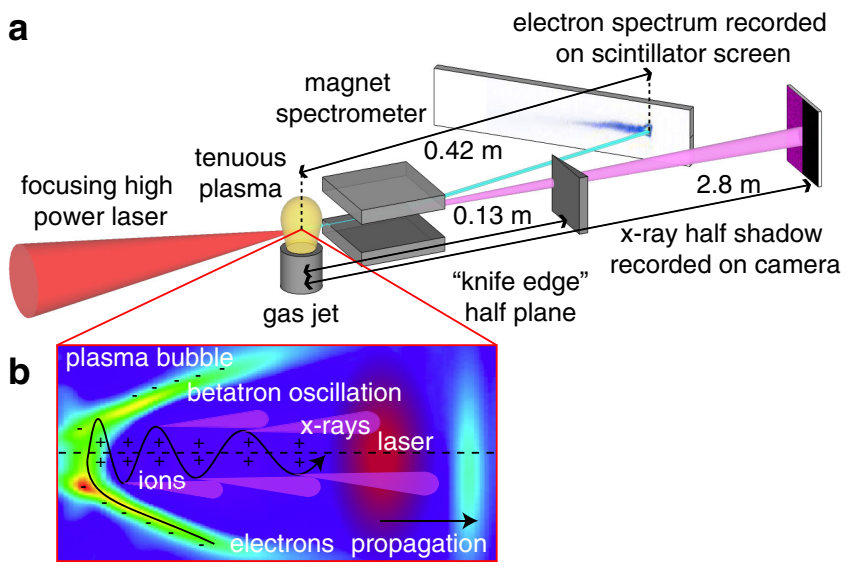

FIG. 1. Schematic of the (a) experimental setup and (b) inside of the plasma wakefield.

in vacuum yielded a FWHM focal diameter of $d_{\mathrm{FWHM}}=$ $(10.8 \pm 0.5) \mu \mathrm{m}$. Typically, $(27 \pm 2) \%$ of the laser energy is within the diameter $d_{\text {FWHM }}$. Helium gas was pulsed through a $3 \mathrm{~mm}$ diameter nozzle creating a supersonic jet. The density profile measured by interferometry has a FWHM length of $(2.7 \pm 0.2) \mathrm{mm}$ and consists of a central plateau, which is constant to within $10 \%$, and linear gradients at the edges.

Figure 1(a) shows a schematic of the experimental setup. A permanent magnet deflects the electrons from the laser axis and disperses them horizontally according to their energy onto a scintillator screen, which is imaged with a CCD camera to obtain the electron spectrum and electron beam divergence. Electrons with energies between 50 and $500 \mathrm{MeV}$ and divergence angles between $\pm 10 \mathrm{mrad}$ can be detected. Electron beams are observed for electron densities between 3 and $8 \times 10^{18} \mathrm{~cm}^{-3}$. A sample electron beam is shown in Fig. 2(a). The average beam charge for 19 shots is found to be $130 \pm 100 \mathrm{pC}$. The vertical electron beam pointing was found to be $0.6 \pm 4.8 \mathrm{mrad}$ about the laser axis. The maximum achievable electron beam energy was found to increase from 70 to $230 \mathrm{MeV}$ for densities from 8 to $3 \times 10^{18} \mathrm{~cm}^{-3}$. Figure 3 shows the scaling of the electron energy with density. At low densities, electron beams were characterized by high energy monoenergetic features, with a low energy dark current [Fig. 2(a)]. For increasing densities, electron beams developed a more complex polyenergetic structure and eventually a $100 \%$ energy spread.

The propagation of the electrons is not affected by the magnet in the direction perpendicular to the dispersion of the spectrometer and thus the vertical electron beam divergence can be obtained from the 1D dispersed signal [Fig. 2(a)]. Since beams are not necessarily monoenergetic, we integrate the dispersed spectrum along the energy axis to obtain an average root mean square (rms) beam divergence. We find that the divergence shows little correlation with the density but may increase somewhat with higher densities. This was observed before [16,17]. 

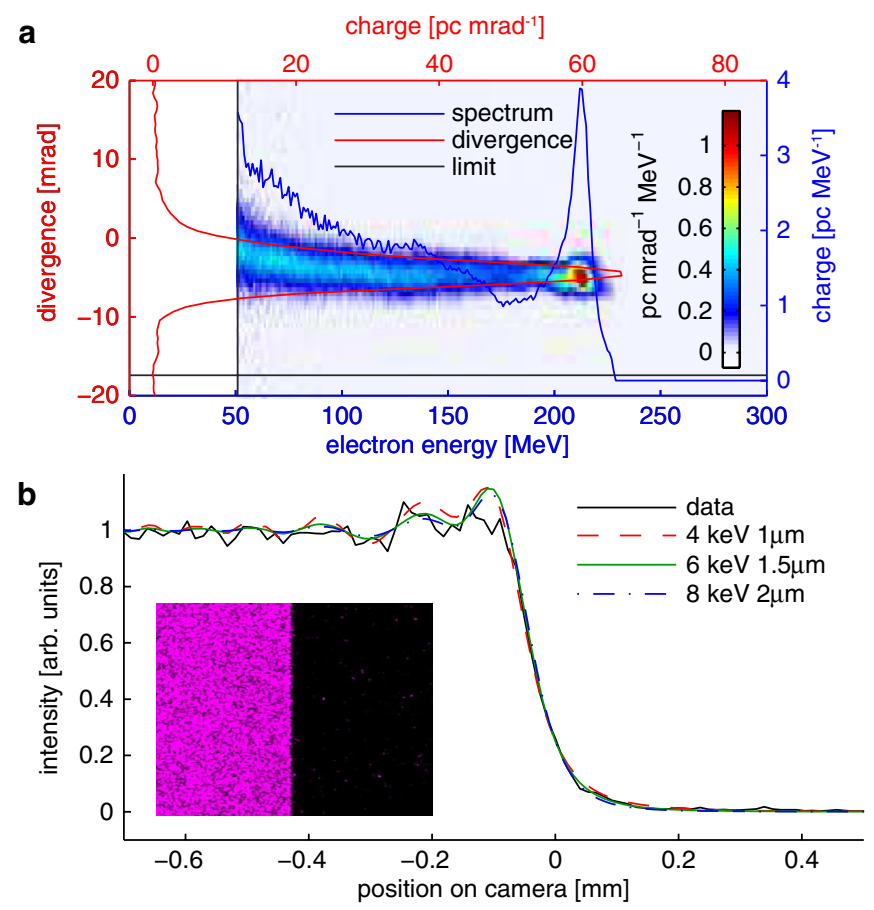

FIG. 2. (a) Sample electron data. Electron charge per mrad and $\mathrm{MeV}$ (2D image), and radially (red) and spectrally (blue) integrated curves. Black lines indicate detector limit and noise level. (b) Sample x-ray half-shadow data (2D image), experimental $\mathrm{x}$-ray intensity integrated along edge (black line), and modeled x-ray intensity using Fresnel diffraction theory (dashed red, full green, dash-dotted blue lines).

It has been shown that electrons accelerated in a plasma wave produce copious amounts of synchrotron-like radiation, termed betatron $\mathrm{x}$ rays $[18,19]$. This is due to the electrons performing transverse oscillations while being accelerated along the laser axis in the fields of the plasma bubble, as illustrated in Fig. 1(b). The characteristics of the betatron $\mathrm{x}$ rays are related to the properties of the electron beam and plasma wiggler. In particular, the small $\mathrm{x}$-ray source size can serve as a diagnostic for the transverse electron bunch size. With our choice of laser parameters, $w_{0}$, the $1 / e^{2}$ spot radius is matched to the bubble radius $w_{0}=r_{b}=2 \sqrt{a} \cdot c / \omega_{p}$ for densities of 3.3-4.9 $\times$ $10^{18} \mathrm{~cm}^{-3}$ and the pulse duration is matched to the bubble radius $c \cdot t_{L}=r_{b}$ for densities of $2.9-4.6 \times 10^{18} \mathrm{~cm}^{-3}$. If these conditions are fulfilled, the wakefield accelerator operates in the bubble regime [4]. Dephasing then coincides with depletion and maximizes the electron beam energy and thus the x-ray power at the end of the interaction. The source size of the $\mathrm{x}$ rays therefore gives the transverse bunch size $r_{\perp}$ of the electron beam at the end of the interaction.

As shown in Fig. 1(a), penumbral imaging is used to determine the betatron x-ray source size. A half-plane (thinly cleaved InSb crystal) is placed after the x-ray source, casting a half shadow on the detector. The experimental intensity distribution integrated along the edge is

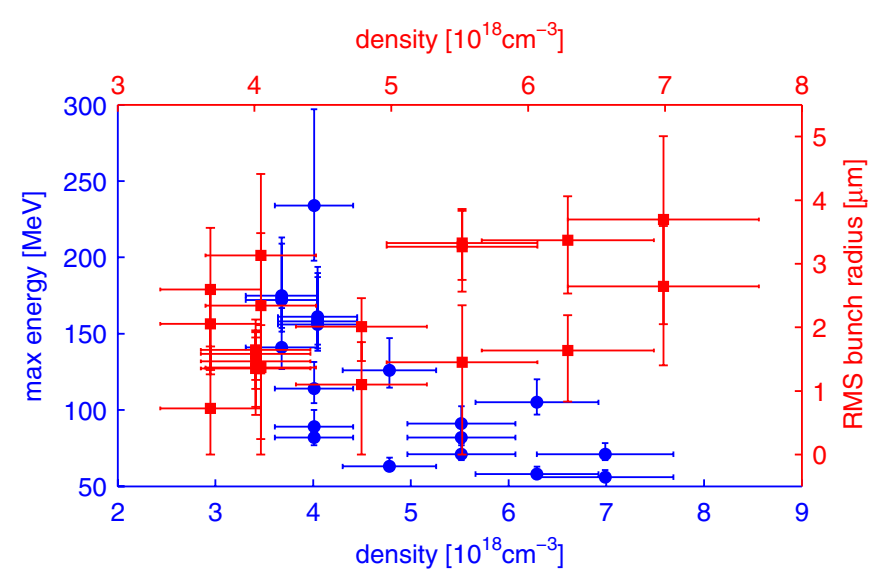

FIG. 3. Maximum electron energy (blue circles, left) and $1 / e^{2}$ electron bunch radius (red squares, right) as a function of density. Horizontal error bars are $\pm 10 \%$ due to uncertainty in density determination and vertical error bars are due to uncertainty in electron beam pointing into spectrometer (4 mrad) and $\mathrm{X}$-ray source size determination (95\% confidence interval [20]). Each data point represents a single shot.

shown in Fig. 2(b) and can be modeled with Fresnel diffraction theory, yielding $\mathrm{x}$-ray spectrum and source size [20]. Using a least-squares method, the rms x-ray source radius (transverse electron bunch radius) is calculated with 95\% confidence interval, as shown in Fig. 3, right axis. Much like the divergence, the x-ray source size also shows little correlation with density.

Using Eqs. (1) and (2), the measured $\gamma, v_{\perp} / v_{\|}$and $r_{\perp}$ can be combined to obtain an upper limit for the rms transverse normalized emittance, which is plotted in Fig. 4. Despite the fact that each measured quantity shows little correlation with density, the emittance gives a correlation of $C=0.84$ with density. The associated $p$ value

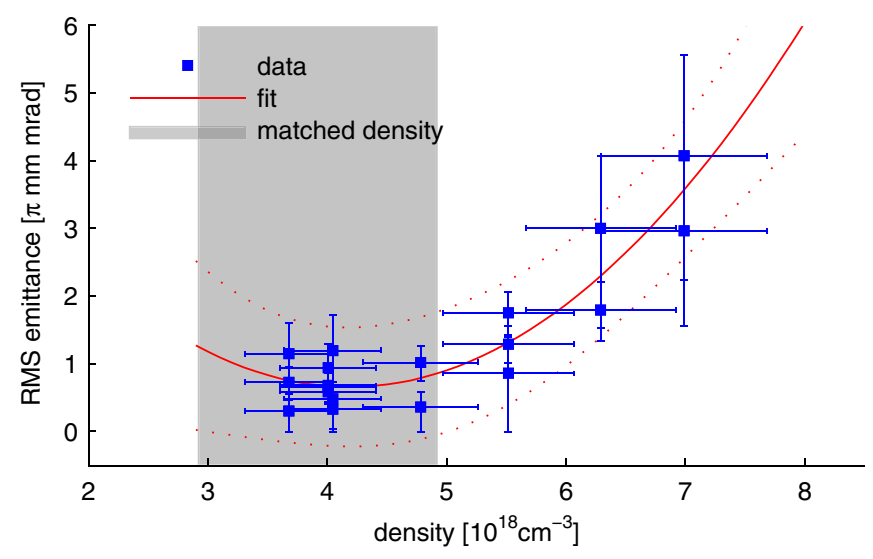

FIG. 4. Experimentally determined emittance as a function of density (blue squares). Fit with a polynomial of 2 nd order (red solid line), giving $R^{2}=0.85$ and $95 \%$ confidence interval (dashed red). The vertical grey lines indicate the density interval for which the spot size $w_{0}=r_{b}$ and pulse duration $c \cdot t_{L}=r_{b}$ matches the bubble radius. Error bars are propagated from errors in x-ray source size and electron energy. 
(probability of getting a correlation as large as the observed value by random chance, when the true correlation is zero) is $P<10^{-4}$. A quadratic fit gives an $R^{2}=0.83$.

Two effects will determine the scaling of the emittance. First, for constant laser pulse duration, the emittance will grow at high densities, as the electron beam is more likely to interact with the laser pulse [16,19]. This should become significant as $c \cdot t_{L}>r_{b}$, i.e., for densities $>2.9-4.6 \times$ $10^{18} \mathrm{~cm}^{-3}$. This trend is reflected in our data (Fig. 4). Second, the emittance will scale with the bubble size $\propto$ $n_{e}^{-1 / 2}$ and the transverse electron momentum spread $\propto a_{0}$ [4], leading to an increase at low densities. This trend is not reflected by our data, which does not extend to densities much lower than where $c \cdot t_{L} \simeq r_{b}$ (shaded area in Fig. 4). The fit to our data however suggests that the emittance is minimized at $n_{e} \simeq 4 \times 10^{18} \mathrm{~cm}^{-3}$, which is when $w_{0}=r_{b}$ and $c \cdot t_{L}=r_{b}$ are matched.

An analytic derivation for the bubble regime using the rms quantities for $\left(r_{\perp}, p_{\perp}\right)$ gives the rms transverse normalized emittance as $\epsilon_{n}^{\perp}=0.033 a_{0}^{3 / 2}\left(n_{\text {crit }} / n_{e}\right)^{1 / 4} \pi \mathrm{mm} \mathrm{mrad}$, which computes to $\epsilon_{n}^{\perp} \simeq 0.87$ and $1.59 \pi \mathrm{mm} \mathrm{mrad}$ for $n_{e}=4 \times 10^{18} \mathrm{~cm}^{-3}$ and $a=3.2$ and 4.8 , respectively [21]. The latter value is the self-consistent trapping threshold for the given density. The predicted emittance is in good agreement with the measured rms normalized transverse emittance as can be seen from Fig. 4 for $n_{e}=4 \times 10^{18} \mathrm{~cm}^{-3}$.

In computing the measured emittance, we used the spectrally averaged divergence $v_{\perp} / v_{\|}$and radially averaged mean energy $\gamma$. The radiated x-ray power per solid angle is $\propto \gamma^{4}$ which affects the measured x-ray source size and electron bunch size $r_{\perp}$. This may motivate a weighing of the $2 \mathrm{D}$ electron data with $\propto \gamma^{4}$ before computing averaged $v_{\perp} / v_{\|}$and $\gamma$. When done, we find that both the absolute number and density trend of the emittance differs insignificantly from our Fig. 4.

Comprehensive numerical modeling was performed to verify if such small emittances can occur in the bubble regime. Three-dimensional particle in cell simulations with the code OSIRIS [22] were carried out, in which a pulse with experimental parameters $\left(a_{0}=3.0, t_{L}=32 \mathrm{fs}\right.$, $\left.d_{\mathrm{FWHM}}=10.8 \mu \mathrm{m}\right)$ is focused $0.25 \mathrm{~mm}$ into the plasma. The electron plasma density profile increases linearly from zero to $n_{e}=6 \times 10^{18} \mathrm{~cm}^{-3}$ in the first $0.5 \mathrm{~mm}$, it is constant for $2.7 \mathrm{~mm}$, and falls linearly to zero in $0.5 \mathrm{~mm}$. To save computational time, simulations were performed in a relativistically boosted frame $(\gamma=5)$ [23]. The simulation box corresponds to $3.5 \times 0.11 \times 0.11 \mathrm{~mm}^{3}$ in the laboratory frame. A total of $9 \times 10^{9}$ particles were pushed for $\approx 6 \times 10^{3}$ iterations. The resolution in the laser propagation direction (in the boosted frame) is $k_{0} \Delta z=0.12$ and $k_{p} \Delta r_{\perp}=0.16$ in the transverse directions. The modeling predicts a polyenergetic electron beam with peaks at 210 and $300 \mathrm{MeV}$, an average energy of $240 \mathrm{MeV}$ and an almost symmetric profile with divergence $v_{\perp} / v_{\|} \simeq 4.6 \mathrm{mrad}$. As

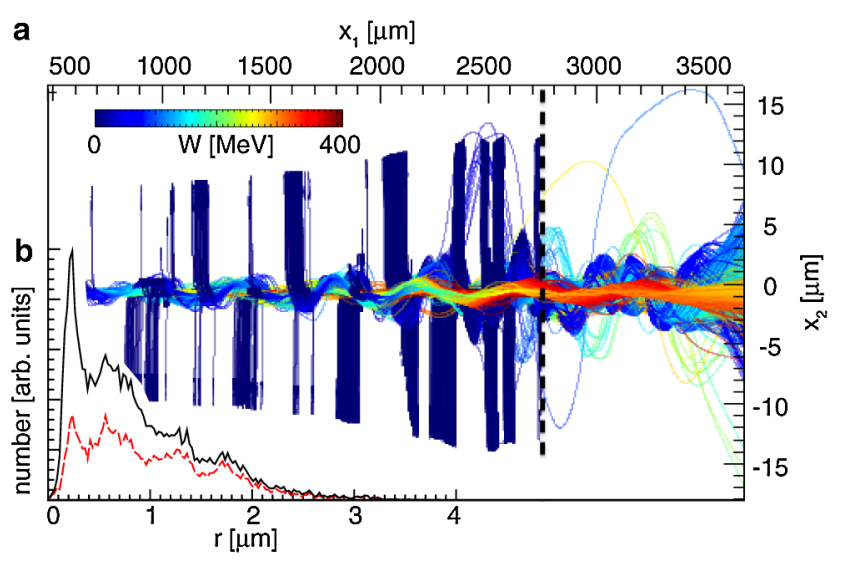

FIG. 5. (a) Electron trajectories obtained from the modeling. (b) Histogram of the betatron amplitudes for entire $3.7 \mathrm{~mm}$ jet (solid black) and for the last $1 \mathrm{~mm}$ (dashed red).

the code tracks the particle trajectories, we find that electrons are injected throughout the interaction and all of them perform oscillating trajectories as depicted in Fig. 5(a). We analyze the oscillation radius for each electron and oscillation to obtain a histogram of oscillation radii, shown in Fig. 5(b). From the histogram we obtain an average oscillation amplitude or bunch radius of $r_{\perp}=0.9$ and $1.0 \mu \mathrm{m}$ respectively, depending on whether we average over the entire length of the trajectory or the last $1 \mathrm{~mm}$. This computes to a transverse normalized emittance of $\epsilon_{n}^{\perp}=0.7 \pi \mathrm{mmmrad}$ for $n_{e}=6 \times 10^{18} \mathrm{~cm}^{-3}$. The same modeling for densities 4 and $4.5 \times 10^{18} \mathrm{~cm}^{-3}$ gives $\epsilon_{n}^{\perp}=0.1 \pi \mathrm{mm} \mathrm{mrad}$. These results agree quantitatively with the measurement and confirm the trend observed in Fig. 4.

For the experimental technique presented in this paper to yield a meaningful (upper) limit of the normalized transverse emittance $\epsilon_{n}^{\perp}$, it needs to be shown that the individual measurements of $\gamma, r_{\perp}$, and $r_{\perp}^{\prime}$ are indeed simultaneous. This means, there must be no magnetic lenses and nothing but drift between the end of the laser-plasma interaction and the planes of the measurement. The source size $r_{\perp}$ is measured at the end of the plateau of the gas density profile, whereas the divergence $r_{\perp}^{\prime}$ and electron energy $\gamma$ are measured after the density down ramp. If the down ramped acted as a lens, this could lead to an increase of the beam size $r_{\perp}$ after the ramp, to which the proposed experimental technique would be blind.

The gravity of possible emittance growth in the down ramp of length $L_{\text {ramp }}$ can be assessed as follows: Electrons oscillating at the betatron period $\lambda_{\beta}$ that transition the density down ramp will feel a reducing transverse restoring force $F \propto n_{e} \cdot r_{\perp}$. Emittance growth can become an issue if $L_{\text {ramp }}$ is no longer small compared to $\lambda_{\beta}$. In our case, for $L_{\text {ramp }}=0.5 \mathrm{~mm}$ and $\lambda_{\beta}=(2 \gamma)^{1 / 2} \lambda_{p}=0.5 \mathrm{~mm}$ for a plasma wavelength $\lambda_{p}=17 \mu \mathrm{m}$ at $4 \times 10^{18} \mathrm{~cm}^{-3}$ and 
$\gamma=460(230 \mathrm{MeV})$. Shorter density down ramps $L_{\text {ramp }}$ can be achieved with more sophisticated gas targets. Operation at lower densities will be accessible with higher power lasers and will increase $\gamma$ and $\lambda_{\beta}$. This means, the demonstrated technique to measure the transverse normalized emittance will be less susceptible to emittance growth in the down ramp for the kind of measurements $(>\mathrm{GeV})$ we propose it for.

To assess if the emittance increases appreciably while the beam travels through the down ramp and plasmavacuum interface, the normalized emittance was also determined directly from the simulation data according to $\epsilon_{n, s}^{\perp}=\langle\gamma \beta\rangle \sqrt{\left\langle r_{\perp}^{2}\right\rangle\left\langle\left(v_{\perp} / v_{\|}\right)^{2}\right\rangle-\left\langle r_{\perp} v_{\perp} / v_{\|}\right\rangle^{2}}[24,25]$ at the end of the plasma density ramp. This confirms that the measurements obtained with $\epsilon_{n}^{\perp}=\gamma r_{\perp} r_{\perp}^{\prime} \geq \epsilon_{n, s}^{\perp}$ are a valid upper limit.

Between plasma-vacuum interface and electron detection plane, there are no further beam line elements that could change the emittance. Space charge effects would only further increase the measured emittance, and it can be concluded that the measurement presented here indeed serves as an upper limit to the normalized transverse emittance of a LWFA.

We have proposed and used a technique to measure the emittance of a LWFA electron beam. The method infers the transverse electron bunch size from the measurement of the betatron $\mathrm{x}$-ray source size. We find the transverse normalized emittance of a $230 \mathrm{MeV}$ electron beam in the bubble regime can be $\epsilon_{n}^{\perp}<0.5 \pi \mathrm{mm}$ mrad similar to conventional accelerators. The method can be applied to multi-GeV electron beams anticipated from near-future LWFA, where standard single shot emittance measurements have limited accuracy and eventually fail. This will be crucial to better diagnose wakefield acceleration.

\section{ACKNOWLEDGMENTS}

This work was supported by U.S. NRC Grant No. 38-09953. The work of J.L.M., R.F., J.V., and L.O.S. is partially supported by FCT (Portugal). The simulations were performed on the IST Cluster (Portugal) and on Jugene (Germany).

[1] T. Tajima et al., Phys. Rev. Lett. 43, 267 (1979).

[2] G. Z. Sun et al., Phys. Fluids 30, 526 (1987).

[3] A. Pukhov et al., Plasma Phys. Controlled Fusion 46, B179 (2004).

[4] W. Lu et al., Phys. Rev. ST Accel. Beams 10, 061301 (2007).

[5] S. P. D. Mangles et al., Nature (London) 431, 535 (2004).

[6] J. Faure et al., Nature (London) 431, 541 (2004).

[7] C. G. R. Geddes et al., Nature (London) 431, 538 (2004).

[8] L.E. Collins et al., Nucl. Instrum. Methods 26, 157 (1964).

[9] Y. Yamazaki et al., Nucl. Instrum. Methods Phys. Res., Sect. A 322, 139 (1992).

[10] S. Fritzler et al., Phys. Rev. Lett. 92, 165006 (2004).

[11] E. Brunetti et al., Phys. Rev. Lett. 105, 215007 (2010).

[12] C. M.S. Sears et al., Phys. Rev. ST Accel. Beams 13, 092803 (2010).

[13] W. P. Leemans et al., Nature Phys. 2, 696 (2006).

[14] S. Kneip et al., Phys. Rev. Lett. 103, 035002 (2009).

[15] K. T. McDonald et al., in Frontiers of Particle Beams: Observation, Diagnosis and Correction, edited by M. Month et al. (Springer, New York, 1989), p. 122.

[16] S.P. D. Mangles et al., Phys. Rev. Lett. 96, 215001 (2006).

[17] S. Kneip et al., Plasma Phys. Controlled Fusion 53, 014008 (2011).

[18] A. Rousse et al., Phys. Rev. Lett. 93, 135005 (2004).

[19] S. Kneip et al., Phys. Rev. Lett. 100, 105006 (2008).

[20] S. Kneip et al., Nature Phys. 6, 980 (2010).

[21] A. G. R. Thomas, Phys. Plasmas 17, 056708 (2010).

[22] R. A. Fonseca et al., Lecture Notes in Computer Science (Springer, Heidelberg, 2002), Vol. 2331, p. 342.

[23] S. F. Martins et al., Nature Phys. 6, 311 (2010).

[24] H. Wiedemann et al., Particle Accelerator Physics (Springer, Heidelberg, 2007).

[25] N. Kirby et al., Phys. Rev. ST Accel. Beams 12, 051302 (2009). 